\title{
Piezoresistive Force Sensor Utilizing Combination of Three-Axis Stress Components
}

\author{
Student member \\ Non-member \\ Member \\ Member
}

\author{
Kazuya Sawa \\ Masato Arai \\ Toshiyuki Toriyama \\ Susumu Sugiyama
}

\author{
(Ritsumeikan University) \\ (Ritsumeikan University) \\ (Ritsumeikan University) \\ (Ritsumeikan University)
}

\section{Summary}

A high sensitive miniature piezoresistive force sensor utilizing combination of three-axis stress components has been developed. This work aims to develop a force sensor which acts as a sensing unit assembled in a new miniature high pressure sensor. By using a force transmission rod, forces are applied to piezoresistors on a Si diaphragm of the sensor. Resistance changes of the piezoresistors depend on combination of stress components generated by the applying forces. FEM stress analysis was carried out, in order to investigate dependence of stress components on dimensions and Young's modulus of the rod, and diaphragm thickness. A sensor structure to obtain large resistance changes of the piezoresistors was found by utilizing valid combination of three-axis stress components according to the analysis. As an experimental result, an output voltage more than $140 \mathrm{mV} / 5 \mathrm{~V}$ at $150 \mathrm{~N}$ was obtained.

Keywords : force sensor, pressure sensor, piezoresistive effect, Si diaphragm, FEM analysis

\section{INTRODUCTION}

Piezoresistive semiconductor pressure sensors have been widely used in the fields of industrial measurement, automobiles, medicine and so on, since they are miniature, light and highly reliable [1-5]. Conventionally, as a typical structure of the piezoresistive $\mathrm{Si}$ pressure sensor, a thin diaphragm is formed on a $\mathrm{Si}$ substrate and piezoresistors are arranged on the diaphragm [6]. However, the thin diaphragm pressure sensor is not suitable for measuring high pressure because of the strength restriction of the diaphragm. Several high pressure sensors have been developed. Generally, oil-sealing type pressure sensors or sensors with piezoresistors bonded on a metal diaphragm are used for measuring high pressure [7]. The oil-sealing type pressure sensors are difficult to compensate temperature characteristic due to thermal expansion of sealed oil. Fabrication process of oil-sealing type sensors are complex and cost are relatively high by introducing oil sealing technology. The sensors with piezoresistors bonded on the metal diaphragm are affected by creep due to bonding portion. It is difficult to bond the small piezoresistors on the metal diaphragm and connect piezoresistors by wires. The structure and fabrication process of the sensors with piezoresistors bonded on a metal diaphragm have been also complex, and it has been difficult to reduce cost and size of the sensors.

It is expected to develop low cost, miniature and

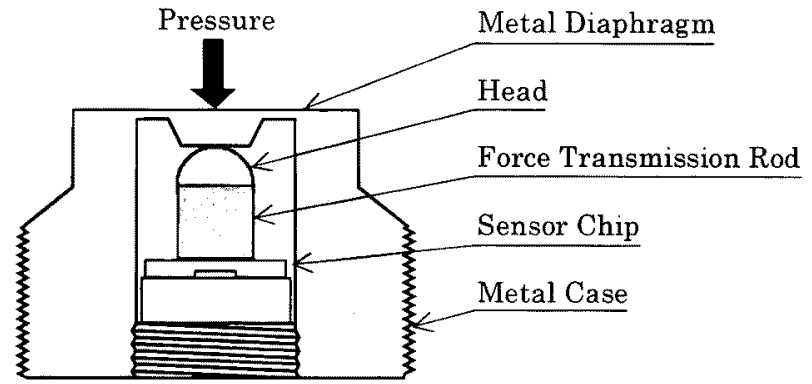

Fig.1 Schematic structure of the pressure sensor.

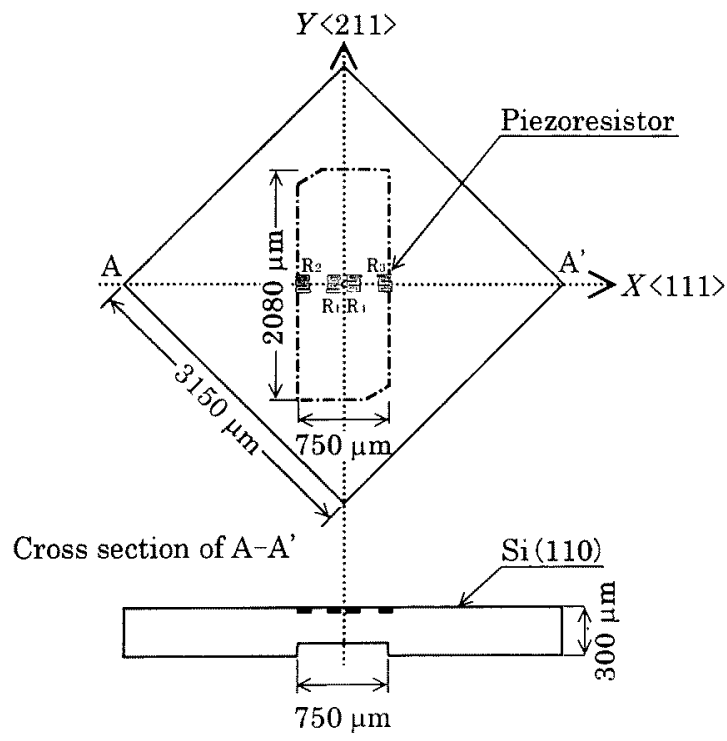

Fig. 2 Schematic of the sensor chip. 
simple structure high pressure sensors in order to measure internal high pressure distribution such as in injection molding machines. For this purpose a force sensor which acts as a sensing unit assembled in a metal diaphragm type high pressure sensor has been developed. The force sensor can measure various range of pressure by changing metal diaphragm thickness of the pressure sensor. Figure 1 shows a schematic structure of the pressure sensor. A pressure applied to a metal diaphragm is transmitted to a sensor chip by using a force transmission rod. Diffused piezoresistors are used as force detecting elements. In this paper, high sensitivity of the force sensor was achieved by utilizing a combination of three-axis stress components on the diaphragm which influence the resistance changes of the piezoresistors. The relation between parameters of the force sensor structure and the output characteristics was investigated by FEM stress analysis. The output characteristics of a prototyped force sensor were reported.

\section{PRINCIPLE}

\subsection{Sensor Structure and Resistance Changes}

A sensor chip as shown in Fig. 2 was adopted as a detecting element of the force sensor. A substrate of the sensor chip was n-type (110) Si. A rectangular diaphragm of $2080 \times 750 \mu \mathrm{m}^{2}$ was formed by using anisotropic etching. Four p-type piezoresistors were formed by impurity diffusion of boron on the surface of diaphragm. A shape and dimension of the piezoresistor is shown in Fig. 3. A structure of the force sensor is shown in Fig. 4. The force sensor was composed of a hemispheric head, a force transmission rod, a sensor chip and a metal plate. The principle of detection is as follows. An elastic deformation of the force transmission rod due to an external applied force is acted on the diaphragm. The resistance changes of the piezoresistors are induced by the stresses of the diaphragm surface. When the stress is applied to the piezoresistors, a resistance change ratio $\Delta R / R$ is expressed by using the piezoresistive effect as follows [8-10].

$$
\begin{aligned}
\Delta R / R=\pi_{11}^{\prime} \sigma_{1}+\pi_{12}^{\prime} \sigma_{2}+\pi_{13}^{\prime} \sigma_{3} \\
+\pi^{\prime}{ }_{14} \sigma_{4}+\pi_{15}^{\prime} \sigma_{5}+\pi_{16}^{\prime} \sigma_{6}
\end{aligned}
$$

Where, $\sigma_{1}, \sigma_{2}$ and $\sigma_{3}$ are the normal stress components. $\sigma_{4}, \sigma_{5}$ and $\sigma_{6}$ are the shearing stress components. $\pi_{11}^{\prime}$, $\cdots, \pi$ ' ${ }_{16}$ are the piezoresistive coefficients with respect to arbitrary directions. $\quad \pi_{11}^{\prime}, \cdots, \pi_{16}^{\prime}$ are able to express in terms of fundamental piezoresistive coefficients $\pi_{11}$, $\pi_{12}$ and $\pi_{44}$. For the p-type piezoresistors, the absolute value of $\pi_{44}$ is much larger than that of $\pi_{11}$ and $\pi_{12}$ [9]. Therefore, Eq. (1) is able to be approximated in

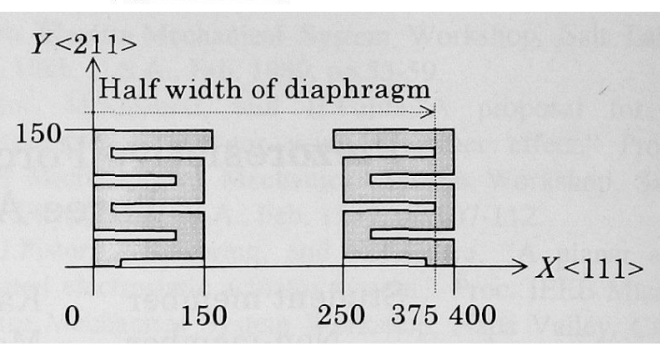

Fig. 3 Dimension of the piezoresistors $(\mu \mathrm{m})$.

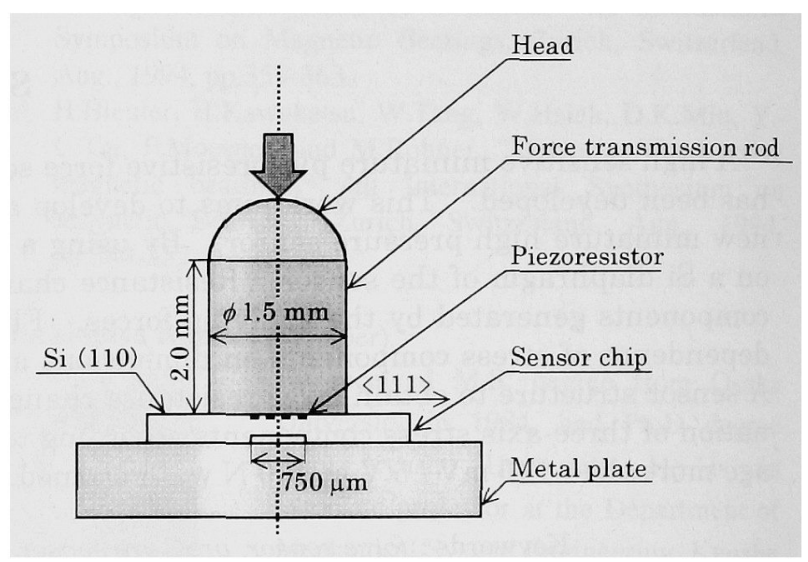

Fig. 4 Structure of the force sensor.

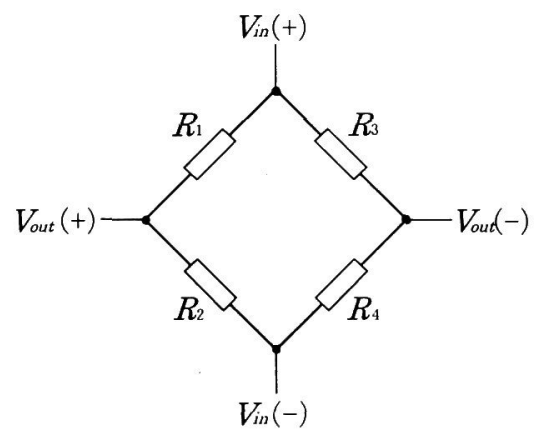

Fig. 5 Full-bridge circuit.

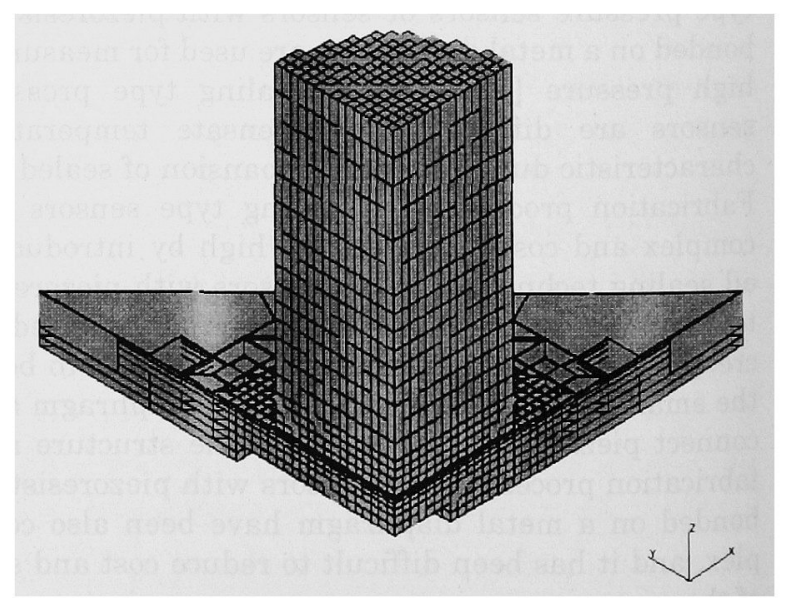

Fig. 6 Analytical model. 
terms of $\pi_{44}$ alone. When $\left.<111\right\rangle$ is selected for longitudinal direction $(X)$ and $<211>$ is selected for transverse direction $(Y)$ as shown in Fig. 2, each piezoresistive coefficients in Eq. (1) are expressed as follows.

$$
\begin{aligned}
& \pi_{11}^{\prime}=\frac{2}{3} \pi_{44} \\
& \pi_{12}^{\prime}=-\frac{1}{3} \pi_{44} \\
& \pi_{13}^{\prime}=-\frac{1}{3} \pi_{44} \\
& \pi_{14}^{\prime}=\pi^{\prime}{ }_{15}=\pi_{16}^{\prime}=0
\end{aligned}
$$

The relation between the stresses and the resistance changes of piezoresistors can be approximately expressed as Eq. (3).

$$
\begin{aligned}
\frac{\Delta R}{R} & \div \frac{2}{3} \pi_{44} \sigma_{1}-\frac{1}{3} \pi_{44} \sigma_{2}-\frac{1}{3} \pi_{44} \sigma_{3} \\
& \fallingdotseq \frac{1}{3} \pi_{44}\left(2 \sigma_{1}-\sigma_{2}-\sigma_{3}\right)
\end{aligned}
$$

Each piezoresistors are arranged into a full-bridge circuit as shown in Fig. 5. According to the circuit, the resistance changes of piezoresistors are transformed into the voltage changes as Eq. (4).

$$
V_{\text {out }}=\frac{1}{2}\left(\frac{\Delta R_{p}}{R_{p}}-\frac{\Delta R_{c}}{R_{c}}\right) V_{i m}
$$

Where, $V_{\text {out }}$ is the output voltage, $V_{\text {in }}$ is the input voltage, $R_{c}$ is the resistance for the central piezoresistors on the diaphragm $\left(R_{1}\right.$ and $\left.R_{4}\right)$ and $R_{s}$ is the resistance for the perimeter piezoresistors on the diaphragm $\left(R_{2}\right.$ and $R_{3}$ ).

\subsection{Combination of Stress Components}

In the case of the ordinary diaphragm pressure sensor, when the pressure is applied on the surface of the sensor chip, stress components generated on the diaphragm can be estimated only or and $\sigma_{2}$, because the other stress components are almost negligibly small. In this case, $\sigma_{1}$ and $\sigma_{2}$ on the center of the diaphragm are negative values (compressive stress), $\sigma_{1}$ and $\sigma_{2}$ on the perimeter of the diaphragm are positive values (tensile stress). $\sigma$ and $\sigma 2$ of both the center and the perimeter have the same signs. Therefore, the resistance changes of the central and the perimeter piezoresistors are proportional to $\left|2 \sigma_{1}-\sigma_{2}\right|$ according to Eq. (3). When the sign of $\sigma_{1}$ is opposite to that of $\sigma_{2}$ and $\sigma_{3}$, larger resistance changes of the piezoresistors is obtained, because the stress term in Eq. (3) becomes summational as $\left|2 \sigma_{1}\right|+\left|\sigma_{2}\right|+\left|\sigma_{3}\right|$. The sensor structure which satisfies the above mentioned combination of stress components is investigated in the following chapter.

\section{FEM ANALYSIS}

\subsection{Analytical Model}

In order to estimate the output characteristics and obtain the most suitable structural parameters of the force sensor, FEM analysis was carried out. An analytical model was generated by MENTAT II, and MARC was used for the analysis [11]. This FEM analysis was three dimensional linear elastic analysis. The analytical conditions were as follows.

- Analytical model

The analytical model was composed of the sensor chip and the force transmission rod. The meshes of the piezoresistor portions having $150 \mu \mathrm{m}$ length were divided with $50 \mu \mathrm{m}$ step in order to obtain more than three nodes on the piezoresistors. The average values of stress of each nodes were used as stresses applied to the piezoresistors. The analysis was carried out by using one fourth part of the model, because of its symmetry. The analytical model is shown in Fig. 6.

- Boundary conditions

All nodes on the bottom of the sensor chip were restricted in $X, Y$ and $Z$ directions, respectively. Since the model was one fourth, the nodes on $X$-axis were restricted in $Y$ direction and the nodes on $Y$ axis were restricted in $X$ direction.

- Load condition

$37.5 \mathrm{~N}$ (one fourth of $150 \mathrm{~N}$ which corresponds to $150 \mathrm{MPa}$ ) was applied to the center of the top of the rod.

\section{- Material properties}

In order to simplify the analysis, Si was assumed to be isotropic material. Young's modulus of Si was $170 \mathrm{GPa}$ and Poisson's ratio was 0.25 [12].

The factors which influence on the output characteristics of the force sensor were considered as follows.

(1) Young's modulus of the rod $(E)$

(2) Height of the rod (h)

(3) Diameter of the rod (d)

(4) Thickness of the diaphragm ( $t)$

As a result of the analysis and the investigation, a basic model was designed as follows : $E=70 \mathrm{GPa}, h=$ $2.0 \mathrm{~mm}, d=1.5 \mathrm{~mm}, t=200 \mu \mathrm{m}$. The variation of stress distributions on the piezoresistors were investigated by changing each factors in (1)-(4) from those of the basic model.

\subsection{Analytical Results}

As examples of analytical results, the distributions 
of each stress components on $X$-axis with the variation of Young's modulus are shown in Fig. 7. As shown in Fig. 7, stress concentration is occurred at $750 \mu \mathrm{m}$ from center due to notch effect of edge of the rod. The impurity concentration was approximately $2 \times 10^{20}$ $\mathrm{cm}^{-3}$. The value of $\pi_{44}=100 \times 10^{-12} \mathrm{~cm}^{2} /$ dyn was used for calculation [13]. A dependence of the resistance changes on the Young's modulus is shown in Fig. 8. A dependence of the resistance changes on the height of the rod is shown in Fig. 9. A dependence of the resistance changes on the diameter of the rod is shown in Fig. 10. A dependence of the resistance changes on the thickness of the diaphragm is shown in Fig. 11.

\subsection{Discussion}

(1) Young's modulus of the $\operatorname{rod}(E)$

The sensor is more and more sensitive as $E$ is smaller as shown in Fig. 8. For the central piezoresistors, the dependence of $\sigma_{1}$ on $E$ was larger than that of $\sigma_{2}$ and $\sigma_{3}$ as shown in Fig. 7. Therefore, $\sigma_{1}$ was most influential for the resistance changes. Duralumin was selected for the rod, because duralumin has smaller $E$ comparing with Si, it is excellent as an elastic material, and it is stable and easy to fabricate.

(2) Height of the rod $(h)$

If $h$ is more than $1.8 \mathrm{~mm}$, the resistance changes were saturated as shown in Fig. 9. Therefore more than $1.8 \mathrm{~mm}$ was desirable to obtain the stable output. $h=2.0 \mathrm{~mm}$ was selected as the height of the rod.

(3) Diameter of the rod (d)

The sensor is more and more sensitive as $d$ is smaller as shown in Fig. 10. With decreasing $d$, the location of peak stress at the rod edge became close to the location of the perimeter piezoresistors and the stress gradients of the perimeter piezoresistors became larger. Therefore, the scatter of output characteristic due to alignment errors might occur. $d=1.5 \mathrm{~mm}$ was selected from the view point of endurance load of sensor chip. When $150 \mathrm{~N}$ as full scale load is applied to the rod having $d=1.5$ $\mathrm{mm}$, the maximum compressive stress of the sensor chip becomes less than half of the compressive fracture stress of $\mathrm{Si}$.

(4) Thickness of the diaphragm $(t)$

In case of less than $t=100 \mu \mathrm{m}, \sigma_{1}, \sigma_{2}$ and $\sigma_{3}$ were approached to zero as shown in Fig. 11. Thus, the resistance changes were also approached to zero. For the perimeter piezoresistors, the resistance changes of $t=150 \mu \mathrm{m}$ was little larger than that of $t$ $=200 \mu \mathrm{m}$ and the largest value was obtained in case of $t=100 \mu \mathrm{m}$. As a result, resistance changes of the piezoresistors were almost same values in the range between $t=150 \mu \mathrm{m}$ and $t=200 \mu \mathrm{m} . \quad t=200$ $\mu \mathrm{m}$ has shortest etching time and highest sensor chip strength against compressive load among this range. Therefore, $t=200 \mu \mathrm{m}$ was selected.

\section{- Thickness of the Si substrate}

The dependence of resistance change on thickness of Si substrate was confirmed by FEM analysis. A Si substrate of $300 \mu \mathrm{m}$ thickness is defined as standard thickness. When the Si substrate thickness changed $\pm 50 \mu \mathrm{m}$ from $300 \mu \mathrm{m}$ of standard thickness, the value of $\left|\sigma_{3}\right|$ on the perimeter piezoresistor changed $\mp 3 \%$. As the result, the sensor output changed $\mp 3 \%$.

\section{CHARACTERISTICS}

\subsection{Prototyped Force Sensor}

The thickness of the diaphragm was $200 \mu \mathrm{m}$. The parameters of the force transmission rod were as follows : $E=70 \mathrm{GPa}, h=2.0 \mathrm{~mm}, d=1.5 \mathrm{~mm}$. The materials of the rod and the metal plate were duralumin and stainless steel, respectively. An epoxy bonding agent was used to bond each part. The above force sensor was fabricated and measured its characteristics.

\subsection{Experimental Results}

The compressive loads to $150 \mathrm{~N}$ were applied to the force transmission rod with Instron (the universal testing instrument). Then the output characteristic of the full-bridge circuit at the input voltage of $5 \mathrm{~V}$ was measured. The typical results are shown in Fig. 12 and Fig. 13.

\subsection{Discussion}

The measured resistance changes of the piezoresistors were negative values at the center $\left(R_{1}, R_{4}\right)$ and positive values at the perimeter $\left(R_{2}, R_{3}\right)$. At $150 \mathrm{~N}$, $\Delta R / R$ of the center was $-2.41 \%$ and that of the perimeter was $2.36 \%$. The correlation between the experimental values and the analytical values were obtained as follows : $\Delta R / R$ of the center was $111 \%$ and that of the perimeter was $91 \%$ comparing with the analytical values. Experimental results were in good agreement with analytical values. As shown in Fig. 12, differences between each pairs of $\Delta R / R$ were due to alignment error of the rod and non-uniform stress distribution which depends on degree of parallel and surface roughness on contact area between the rod and the sensor chip. In fabrication of the prototyped force sensor, alignment error was within $\pm 20 \mu \mathrm{m}$. FEM analysis revealed that when alignment error is within $\pm 20 \mu \mathrm{m}$, change of $\Delta R / R$ on central piezoresistor is from $-19.0 \%$ to $+8.2 \%$, and that of on perimeter 


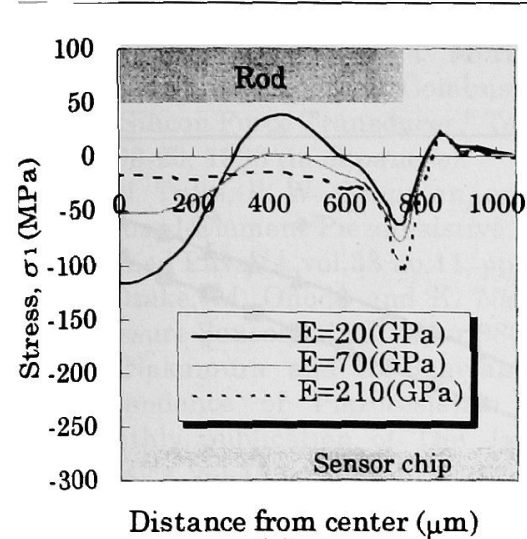

(a)

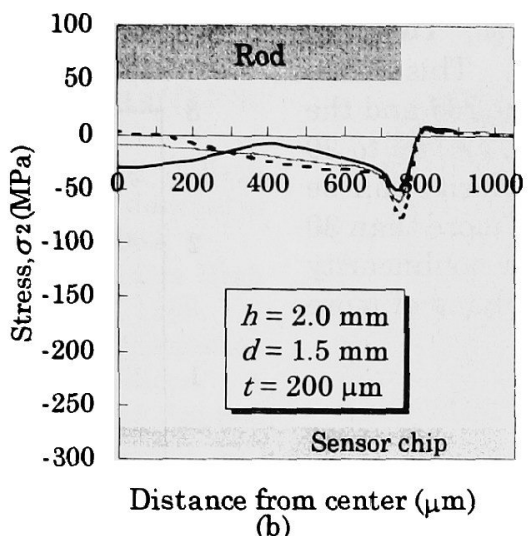

(b)

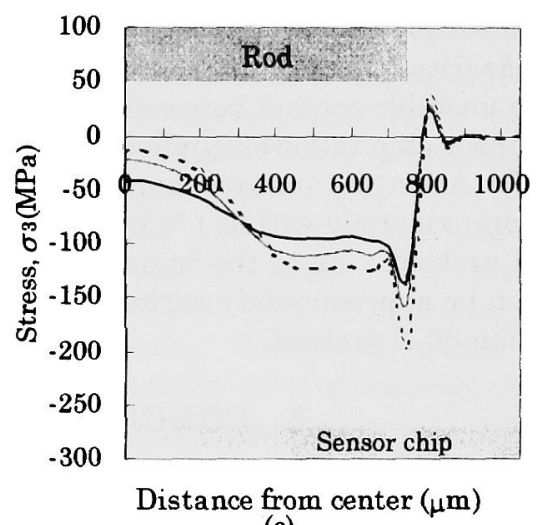

(c)

Fig. 7 Dependence of stress distribution on Young's modulus $(E)$.

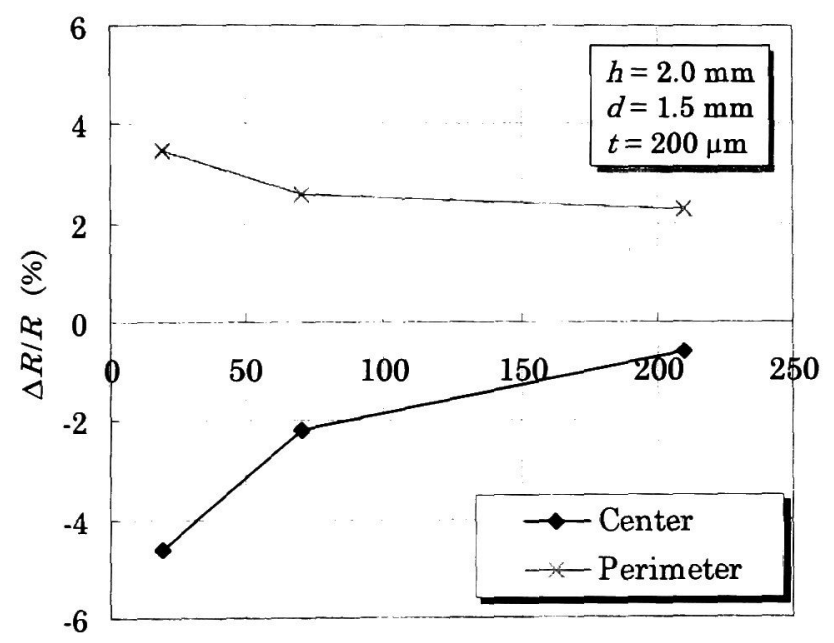

Young's modulus of rod, $E(\mathrm{GPa})$

Fig. 8 Dependence of resistance changes on Young's modulus $(E)$.

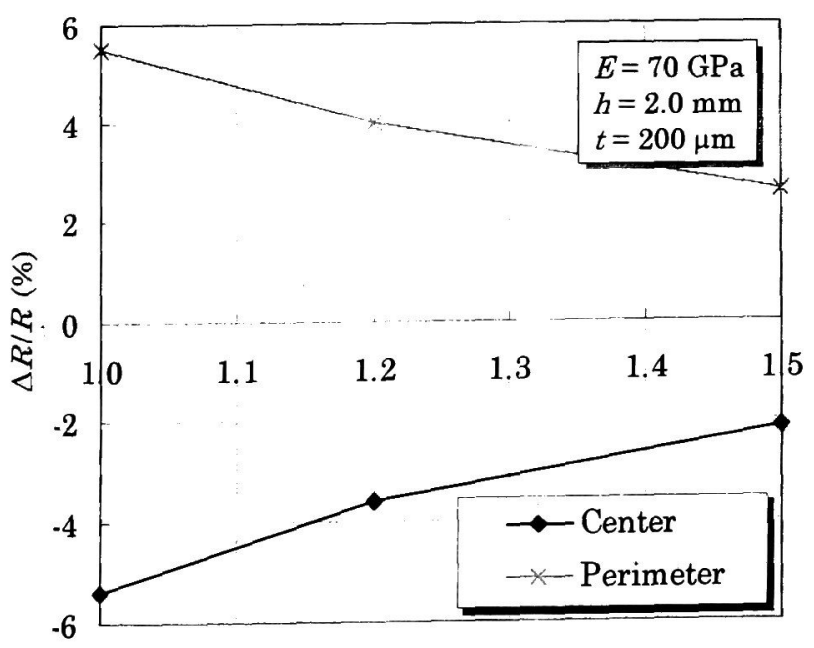

Diameter of rod, $d(\mathrm{~mm})$

Fig. 10 Dependence of resistance changes on diameter of rod $(d)$.

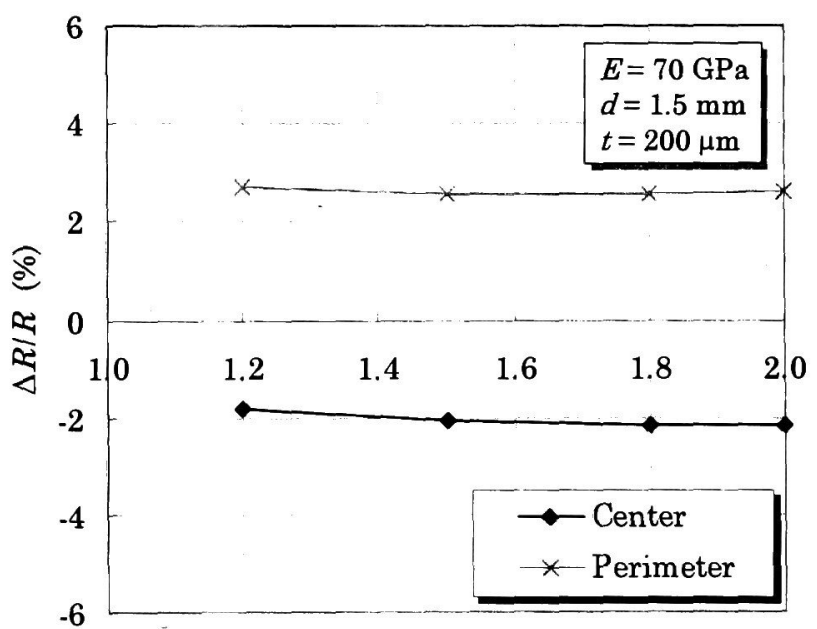

Height of rod, $h$ (mm)

Fig. 9 Dependence of resistance changes on height of $\operatorname{rod}(h)$.

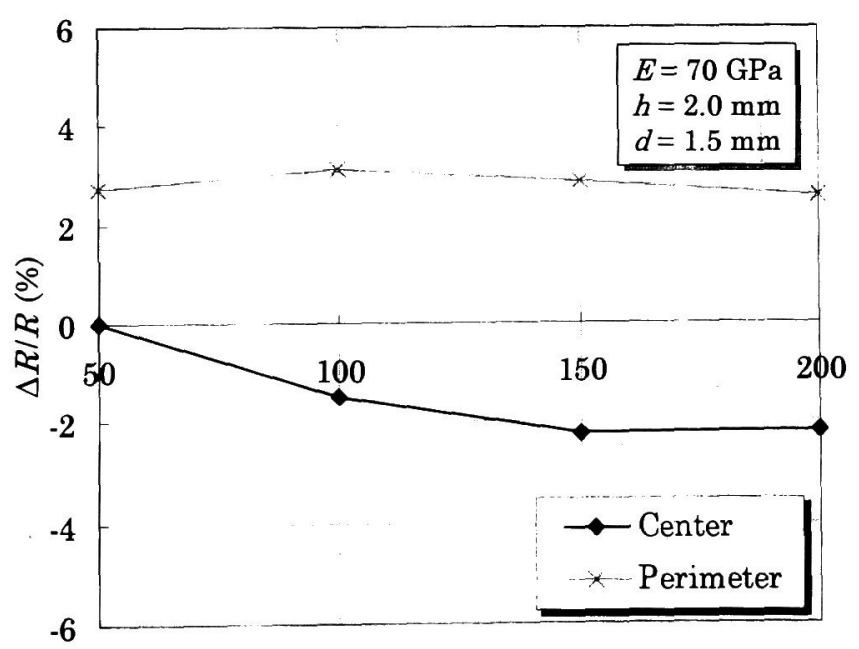

Thickness of diaphragm, $t(\mu \mathrm{m})$

Fig. 11 Dependence of resistance changes on thickness of diaphragm $(t)$. 
piezoresistor is from $-20.0 \%$ to $+16.0 \%$. The nonlinearity of $5.1 \%$ (F.S.) wasn't too much. This is due to unstable contact between the head, the rod and the sensor chip in lower applied load region, i.e., up to 30 N. As to the improvement, the nonlinearity can be approximately within $1 \%$ by applying of more than 30 $\mathrm{N}$ preload. As to the improvement, the nonlinearity can be approximately within $1 \%$ by applying of more than $30 \mathrm{~N}$ preload.

\section{CONCLUSIONS}

A structure of a piezoresistive force sensor unit which will be assembled into a miniature high pressure sensor with high sensitivity was investigated. In order to obtain relations between parameters of the force sensor structure and output characteristics, FEM analysis was carried out. The sensor structure to obtain large resistance changes of the piezoresistors was found by utilizing valid combination of three-axis stress components. According to FEM analytical results, duralumin of Young's modulus $E=70 \mathrm{GPa}$ was selected as a material of the force transmission $\operatorname{rod}(h=2.0 \mathrm{~mm}, d=1.5 \mathrm{~mm})$. As an experimental result, the output voltage more than $140 \mathrm{mV} / 5 \mathrm{~V}$ at $150 \mathrm{~N}$ was obtained. In the future work, we will improve the precision of fabrication and develop the high pressure sensor assembled from the force sensor.

\section{ACKNOWLEDGEMENTS}

The authors would like to thank Mr. K. Akagi, Mr. Y. Ota and Mr. T. Hatasaki at RIKEN KEIKI CO. ,LTD. for fabrications, measurements and discussions of the force sensors, Mr. H. Iwata and Mr. K. Itoigawa at TOKAI RIKA CO. ,LTD. for supplying sensor chips, and Mr. Y. Nasu and Dr. T. Imamichi at Industrial Research Center of Shiga Prefecture for the helping of measurements of force sensors.

(Manuscript received May 7, 1998, revised Feb. 12, 1999)

\section{REFERENCES}

[1]S. Sugiyama, H. Nakamura, and I. Igarashi, "Mechanical-Electrical Semiconductor Transducers," Instrumentation and Automation, vol.3 no.5, 1975 (in Japanese).

[2]S. Sugiyama and I. Igarashi, "Thin Semiconductor Load Cell," Journal of JSNDI, vol.26 no.8, pp.516-520, 1977 (in Japanese).

[3]S. Sugiyama and O. Tabata, "Evaluation of Thin Film Mechanical Properties for Micromechanism," Journal of the Society of Instrument and Control Engineers, vol.28 no.6, pp.485-488, 1989 (in Japanese).

[4]Joseph Garvey, David Beebe, and Denice D. Denton, "Finite Element Modeling of a Silicon Tactile Sensor," Sensor and Materials, vol.9 no.4, pp.197-213, 1997.

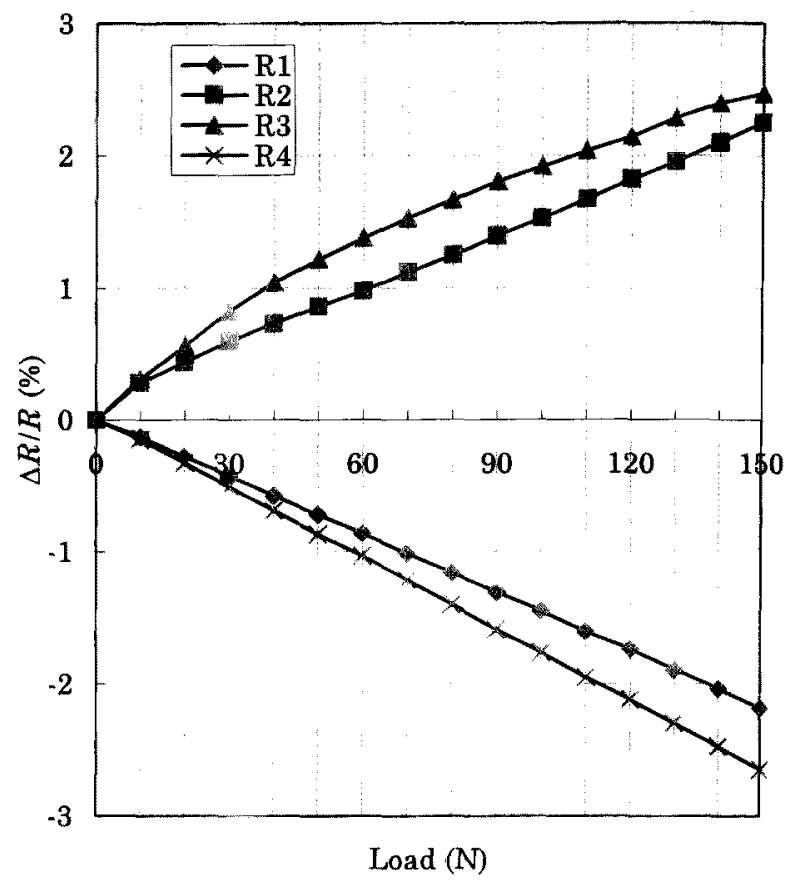

Fig. 12 Resistance changes of piezoresistors versus applied load.

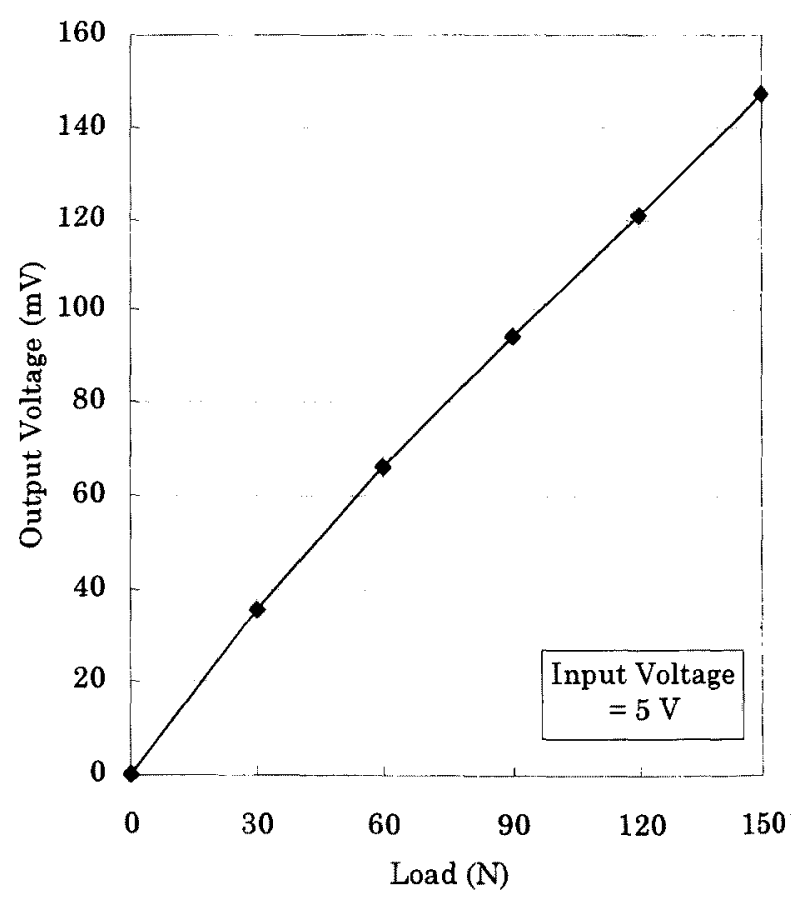

Fig. 13 Output characteristics of the force sensor. 
[5]Y. Omura, K. Tsukada, T. Morikawa, Y. Nonomura, H. Goto and A. Hosono, "A Combustion Pressure Sensor using Silicon Force Transducer," Technical Report of IEICE, ED93-20, 1993 (in Japanese).

[6]O. N. Tufte, P. W. Chapman, and Donald Long, "Silicon Diffused-Element Piezoresistive Diaphragms," Journal of Applied Physics, vol.33 no.11, pp.3322-3327, 1962.

[7]S. Otake, M. Onoda and K. Nagase, "Automotive High Pressure Sensor," SAE Paper,980271,pp.61-68, 1998.

[8]H. Nakamura and S. Sugiyama "Crystal Orientation Dependence of Piezoresistive Effect in Silicon," A monthly publication of The Japan Society of Applied Physics, vol.45 no.2, pp.179-182, 1976 (in Japanese).

[9]C. S. Smith, "Piezoresistance Effect in Germanium and Silicon," Physical Review, vol.94 no.1, pp.42-49, 1954.

[10]W. G. Pfann and R. N. Thuston, "Semiconducting Stress Transducers Utilizing the Transverse and Shear Piezoresistance Effects," Journal of Applied Physics, vol.32 no.10, pp.2008-2019, 1961.

[11]MARC Analysis Research Corporation, "MARC, MENTAT II Command Reference," RF-3008-2.2,2.3, 1995.

[12]J. J. Wortman and R. A. Evans, 'Young's modulus, Shear Modulus, and Poisson's Ratio in silicon and Germanium," Journal of Applied Physics, vol.36 no.1, pp.153-156, 1965.

[13]O. N. Tufte and E. L. Stelzer, "Piezoresistive Properties of Silicon Diffused Layers," Journal of Applied Physics, vol.34 no.2, pp.313-318, 1963.

\section{Kazuya Sawa ( student member )}

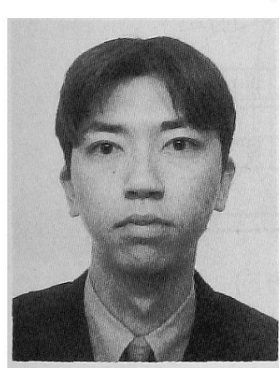

$\mathrm{He}$ received the B.S. degree in 1997, and the M.S. degree in 1999, in Mechanical Engineering from Ritsumeikan University, Shiga, Japan. He joined OMRON Co. in 1999.

\section{Toshiyuki Toriyama ( member)}

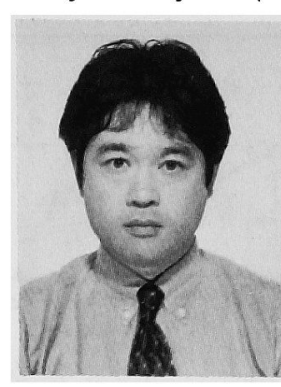

He received the B.S. degree in 1985, the M.S. degree in 1987, in Mechanical Engineering from Ritsumeikan University, Shiga, Japan, and Ph.D. degree in 1994 from Kyushu University, Fukuoka, Japan. He is now a post doctoral fellow in Ritsumeikan University. His current interest is piezoresistive effect of semiconductor material and its application to microsensor.

\section{Susumu Sugiyama ( member)}

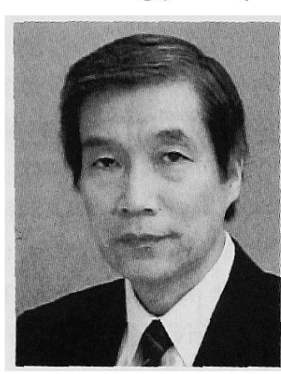

He received the B.S. degree in Electrical Engineering from Meijo University, Nagoya, in 1970, and the Dr. E. degree from Tokyo Institute of Technology, Japan, in 1994. From 1965 to 1995, he was with Toyota Central Research \& Development Laboratories, Inc., where he worked on semiconductor strain gages, silicon pressure sensors, integrated sensors and micromachining. While there, he was a Senior Researcher, Manager of the Silicon Devices Laboratory, and Manager of the Device Development Laboratory. Since 1995 he has been with Ritsumeikan University, Shiga, Japan, where he recently serves as a Professor in the Department of Robotics, Faculty of Science and Engineering. He is Vice Director of Synchrotron Radiation Center at Ritsumeikan University, Editor-in-Chief of Sensors and Materials. His current interests are microsensors and microactuators and high aspect ratio micro structure technology. $\mathrm{He}$ is a member of the IEEE, Japan Society of Applied Physics and the Robotics Society of Japan.
Masato Arai (non-member )

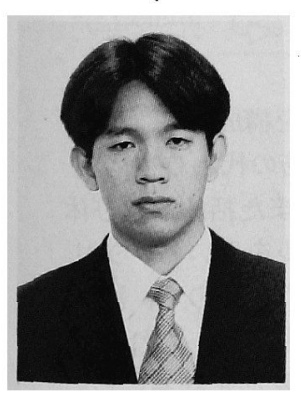

$\mathrm{He}$ received the B.S. degree in 1996, and the M.S. degree in 1998, in Mechanical Engineering from Ritsumeikan University, Shiga, Japan. He joined DENSO Co. in 1998. 